\title{
THE RELATIONSHIP BETWEEN PHYSICAL ACTIVITY AND BREAST CANCER: PREVENTION, TREATMENT AND RECONSTRUCTION OF SELF-ESTEEM
}

Natália S. D. Mendonça', Guilherme A. Sampaio1, Laine R. Martins¹, Júlia de J. Caetano', Murilo Henrique Costa e Silva', Juliana A. Sampaio²

${ }^{1}$ Faculdade de Medicina, Universidade Federal de Goiás - Goiânia (GO), Brazil.

${ }^{2}$ Faculdade de Fisioterapia, Pontifícia Universidade Católica de Goiás - Goiânia (GO), Brazil.

Objective: It is known that a physical activity promotes the balance of hormone levels, strengthens the body's defenses, and helps the maintenance of adequate body weight. Therefore, it contributes to prevent various types of cancer, including breast cancer. In this perspective, the present work seeks to understand the relationship between physical activity and Breast Cancer. Methodology: This study was searched on PubMed, SciELO, Lilacs for relevant publications. The keywords used were "breast cancer" AND "exercise” AND "prevention” AND “treatment”. Results: Fatigue on cancer causes decrease in quality of life and in daily functional capacity of patients. To escape more drugs, a physical activity appears with the best benefit to eliminate this symptom and to increase the disposition of the patients. The impact on self-esteem is in the discovery of overcoming. It also helps control weight and reduce swelling caused by the use of corticosteroids in chemotherapy. Group physical activities promote the patient's interaction with other people, facilitating distraction from the disease. About the prevention, according to researches there was a $25 \%$ average risk reduction amongst physically active women as compared to the least active women. The production of estrogen by adipose tissue can be one of the reason: the more fat the body has, the more of that hormone will be produced. The cancer cells use the substance as a fuel, since it acts on the mammary cells. Another hormone is insulin, that induces the multiplication of cells, so once the cancer starts, having high levels of insulin in the blood becomes dangerous, and exercising decrease the amount of that substance in the body. Conclusion: There is no doubt about the importance of practicing physical activities regularly. In addition to contributing to the prevention, treatment and recovery of self-esteem in patients with breast cancer, physical exercises have other health benefits, and their insertion into our routines is indispensable. 